Pacific Journal of Mathematics

PERMANENT OF THE DIRECT PRODUCT OF MATRICES 


\section{PERMANENT OF THE DIRECT PRODUCT OF MATRICES}

\section{RICHARD A. BRUALDI}

Let $A$ and $B$ be nonnegative matrices of orders $m$ and $n$ respectively. In this paper we derive some properties of the permanent of the direct product $A \times B$ of $A$ with $B$. Specifically we prove that

$$
\operatorname{per}(A \times B) \geqq(\operatorname{per}(A))^{n}(\operatorname{per}(B))^{m}
$$

with equality if and only if $A$ or $B$ has at most one nonzero term in its permanent expansion. We also show that every term in the permanent expansion of $A \times B$ is expressible as the product of $n$ terms in the permanent expansion of $A$ and $m$ terms in the permanent expansion of $B$, and conversely. This implies that a minimal positive number $K_{m, n}$ exists such that

$$
\operatorname{per}(A \times B) \leqq K_{m, n}(\operatorname{per}(A))^{n}(\operatorname{per}(B))^{m}
$$

for all nonnegative matrices $A$ and $B$ of orders $m$ and $n$ respectively. A conjecture is given for the value of $K_{m, n}$.

Definitions. Let $A=\left[a_{i j}\right]$ be a matrix of order $m$ with entries from a field $F$. The permanent of $A$ is defined by

$$
\operatorname{per}(A)=\Sigma a_{1 i_{1}} a_{2 i_{2}} \cdots a_{m i_{m}},
$$

where the summation extends over all permutations $\left(i_{1}, i_{2}, \cdots, i_{m}\right)$ of the integers $1,2, \cdots, m$. The set of elements

$$
a_{1 i_{1}}, a_{2 i_{2}}, \cdots, a_{m i_{m}}
$$

where $\left(i_{1}, i_{2}, \cdots, i_{m}\right)$ is a permutation of $1,2, \cdots, m$ is called a permutation array of $A$, while their product

$$
a_{1 i_{1}} a_{2 i_{2}} \cdots a_{m i_{m}}
$$

is a permutation product of $A$. The permanent of $A$ is then the sum of all the permutation products of $A$. The term rank $\rho(A)$ of the matrix $A$ is defined to be the maximal order of a minor of $A$ with a nonzero term in its determinant expansion. By a theorem of König [3] it is also equal to the minimal number of lines (rows and columns) which collectively contain all the nonzero entries of $A$. Obviously $\rho(A)=m$ if and only if $A$ has a nonzero permutation product. A good discussion of these concepts is given by H.J. Ryser in [3].

If $B$ is another matrix of order $n$ with entries from the field $F$, 
then the direct product (or Kronecker product) of $A$ with $B$ is defined by

$$
A \times B=\left[\begin{array}{cccc}
a_{11} B & a_{12} B & \cdots & a_{1 m} B \\
a_{21} B & a_{22} B & \cdots & a_{2 m} B \\
\vdots & \vdots & & \vdots \\
a_{m 1} B & a_{m 2} B & \cdots & a_{m m} B
\end{array}\right] .
$$

It is a matrix of order $m n$. The submatrix of $A \times B$ given by

$$
\left[a_{i j} B\right] \quad(1 \leqq i, j \leqq m)
$$

is called the $(i, j)$-block of $A \times B$ or sometimes simply a block of $A \times B$. Direct products are discussed by C.C. MacDuffee in [2]. We mention those properties which will be of use to us. First it is readily verified that an associative law is satisfied, so that $A_{1} \times A_{2} \times \cdots \times A_{k}$ can be defined unambiguously. If $C$ and $D$ are matrices of orders $m$ and $n$ respectively, then

$$
(A \times B)(C \times D)=A C \times B D .
$$

Thus if $P A P_{1}=A_{1}$ where $P$ and $P_{1}$ are permutation matrices of order $m$ and if $Q B Q_{1}=B_{1}$ where $Q$ and $Q_{1}$ are permutation matrices of order $n$, then

$$
(P \times Q)(A \times B)\left(P_{1} \times Q_{1}\right)=A_{1} \times B_{1} .
$$

This says that permutations of the rows and columns of $A$ and $B$ induce permutations of the rows and columns of $A \times B$.

It follows by inspection that a permutation matrix $P$ of order $m n$ exists such

$$
P^{r}(A \times B) P=B \times A,
$$

where $P^{T}$ denotes the transpose of $P$. That is, the rows and columns of $A \times B$ can be simultaneously permuted to give $B \times A$. From this we immediately obtain

$$
\operatorname{per}(A \times B)=\operatorname{per}(B \times A) .
$$

A formula for the determinant of $A \times B$ is given by

$$
\operatorname{det}(A \times B)=(\operatorname{det}(A))^{n}(\operatorname{det}(B))^{m} .
$$

The definition of the determinant is very similar to that of the permanent, the only difference being that in the determinant we assign a certain sign to the permutation products. It is therefore natural to ask whether (1.5) has a counterpart for the permanent. It is this 
question that we consider for nonnegative matrices $A$ and $B$. A nonnegative matrix is one whose entries are nonnegative real numbers. Such matrices are discussed by Gantmacher in [1].

This paper is taken from a portion of the author's doctoral dissertation submitted to Syracuse University in June, 1964 and written under the supervision of Professor H.J. Ryser. The author wishes to take this opportunity to express his sincere appreciation to Professor Ryser for his excellent guidance. The dissertation was written during a period in which the author held a summer fellowship of the National Science Foundation and a fellowship of the National Aeronautics and Space Administration.

2. Preliminary theorems. A well-known theorem due to Frobenius and König asserts (in our terminology) that all permutation products of $A$ are zero if and only if $A$ contains an $s$ by $t$ submatrix of 0 's with $s+t=m+1, m$ being the order of $A$. We divide the opposite situation where $A$ has at least one nonzero permutation product into two cases.

THEOREM 2.1. Let $A$ be a matrix of order $m$ with entries from a field $F$. Then $A$ has precisely one nonzero permutation product if and only if by permutations of its rows and columns it may be brought to the triangular form:

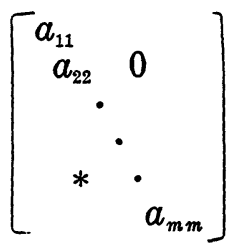

where $a_{11} a_{12} \cdots a_{m m} \neq 0$. In (2.1.), 0 denotes all zeros while * denotes arbitrary elements.

Proof. We need only prove the necessity. Thus suppose $A$ has precisely one nonzero permutation product. If $m=2$, the result is readily verified. We may regard $A$ as the incidence matrix of a collection of subsets $S_{1}, S_{2}, \cdots, S_{m}$ of the elements $x_{1}, x_{2}, \cdots, x_{m}$. Here $x_{j}$ is a member of $S_{i}$ if and only if $a_{i j} \neq 0$. The fact that $A$ has precisely one nonzero permutation product means the subsets $S_{1}, S_{2}, \cdots, S_{m}$ have exactly one system of distinct representatives. If all of the subsets $S_{1}, S_{2}, \cdots, S_{m}$ contained two or more elements, then by [3, Thm. 1.2, p. 48] there would be at least two systems of distinct representatives. Hence one of the subsets $S_{1}, S_{2}, \cdots, S_{m}$ must contain only one element. Therefore by permuting the rows and columns of $A$ we may assume $A$ has the form 


$$
\left[\begin{array}{c|cccc}
a_{11} & 0 & 0 & \cdots & 0 \\
\hline * & & & \\
* & & & \\
\vdots & & & A^{1} \\
* & & &
\end{array}\right]
$$

where $a_{11} \neq 0$ and $*$ denotes arbitrary elements. Now $A^{1}$ can have only one nonzero permutation product otherwise $A$ would have more than one. Applying induction to $A^{1}$, we obtain desired result.

CoRollary 2.2. Let $A$ be a $(0,1)$-matrix of order $m$. Then per $(A)=1$ if and only if the row and columns of $A$ can be permuted to yield a triangular matrix with 1's on the main diagonal and 0's above the main diagonal.

THEOREM 2.3. Let $A$ be a matrix of order $m$ with entries from an arbitrary field $F$. Then $A$ has more than one nonzero permutation product if and only if the rows and columns of $A$ can be permuted to give a configuration of the form:

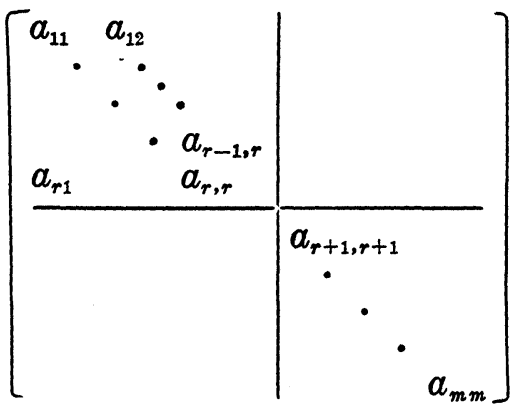

where $2 \leqq r \leqq m$ and each $a_{i j}$ designated above is not zero. All entries not designated are arbitrary.

Proof. ${ }^{1}$ Suppose $A$ has more than one nonzero permutation product. By permuting rows and columns we may assume to begin with that the elements on the main diagonal of $A$ are nonzero. The conclusion now follows by using the well-known fact that if $Q$ is a permutation matrix then there exists another permutation matrix $P$ such that $P^{T} Q P$ is the direct sum of full cycle permutation matrices.

THEOREM 2.4. Let $A$ and $B$ be nonnegative matrices of order $n$. Then

$$
\operatorname{per}(A B) \geqq \operatorname{per}(A) \operatorname{per}(B) \text {. }
$$

\footnotetext{
1 The author is indebted to the referee for improving the exposition here.
} 
Strict inequality occurs in (2.3) if and only if there exists an integer $i$ with $1 \leqq i \leqq n$ having the property that if $A_{i}$ denotes the matrix $A$ with column $i$ deleted and $B_{i}$ denotes the matrix $B$ with row $i$ deleted, then

$$
\operatorname{per}\left(A_{i} B_{i}\right)>0
$$

Proof. Every permutation product of $A B$ is the sum of $n^{n}$ terms, each of which consists of the product of $n$ elements of $A$ and $n$ elements of $B$. Consider a term

$$
a_{1 i_{1}} b_{i_{1} j_{1}} \cdots a_{n i_{n}} b_{i_{n} j_{n}}
$$

of per $(A)$ per $(B)$. Here $\left(i_{1}, \cdots, i_{n}\right)$ and $\left(j_{1}, \cdots, j_{n}\right)$ are permutations of the integers $1, \cdots, n$. The expression (2.5) is a term in the permutation product of $A B$ arising from the elements of $A B$ in positions $\left(1, j_{1}\right), \cdots,\left(1, j_{n}\right)$. From this and the fact that $A$ and $B$ are nonnegative matrices, (2.3) follows.

Strict inequality occurs in (2.3) if and only if some permutation product of $A B$ contains a nonzero term of the form

$$
a_{1 i_{1}} b_{i_{1} j_{1}} \cdots a_{n i_{n}} b_{i_{n} j_{n}}
$$

where $\left(j_{1}, \cdots, j_{n}\right)$ is a permutation of the integers $1, \cdots, n$ and where $1 \leqq i_{s} \leqq n$ for $s=1, \cdots, n$, but $\left(i_{1}, \cdots, i_{n}\right)$ is not a permutation of of $1, \cdots, n$. Thus there exists at least one integer $k$ between 1 and $n$ such that $i_{j}$ is different from $k$ for $j=1, \cdots, n$. Let $A_{k}$ be the matrix obtained by crossing out column $k$ of $A$ and $B_{k}$ the matrix obtained by crossing out row $k$ of $B$. Then a nonzero term of the form (2.6) occurs if and only if per $\left(A_{k} B_{k}\right)>0$. This establishes the theorem.

3. Main theorems. We now prove the main result of this paper.

THEOREM 3.1. Let $A$ and $B$ be nonnegative matrices of orders $m$ and $n$ respectively. Then

$$
\operatorname{per}(A \times B) \geqq(\operatorname{per}(A))^{n}(\operatorname{per}(B))^{m} .
$$

Equality occurs in (3.1) if and only if $A$ or $B$ has at most one nonzero permutation product.

Proof. We have

$$
A \times B=\left(A \times I_{n}\right)\left(I_{m} \times B\right),
$$


where $I_{m}$ and $I_{n}$ are identity matrices of orders $m$ and $n$ respectively. Hence by Theorem 2.4,

$$
\operatorname{per}(A \times B) \geqq \operatorname{per}\left(A \times I_{n}\right) \operatorname{per}\left(I_{m} \times B\right) \text {. }
$$

But

$$
\operatorname{per}\left(I_{m} \times B\right)=(\operatorname{per}(B))^{m},
$$

since $I_{m} \times B$ is the direct sum of $B$ taken $m$ times. Also

$$
\operatorname{per}\left(A \times I_{n}\right)=\operatorname{per}\left(I_{n} \times A\right)=(\operatorname{per}(A))^{n}
$$

by (1.4). This establishes (3.1).

We now investigate the circumstance of equality in (3.1). We remark that equality occurs in (3.1) is and only if equality occurs in (3.2). Necessary and sufficient conditions that equality occur in (3.2) are given in Theorem 2.4. In proving that equality occurs under the conditions stated in the theorem we may assume by (1.4) that $A$ has at most one nonzero permutation product. If all permutation products of $A$ are zero, then per $(A)=0$ and the term $\operatorname{rank} \rho(A)$ of $A$ satisfies $\rho(A)<m$. It then follows by an easy application of König's Theorem that

$$
\rho(A \times B) \leqq \rho(A) n<m n .
$$

Therefore $\operatorname{per}(A \times B)=0$, and equality occurs in (3.1). It $A$ has precisely one nonzero permutation product, then according to Theorem 2.1 the rows and columns of $A$ can be permuted to give the triangular matrix

$$
\left[\begin{array}{cc}
a_{11} & \\
\bullet & 0 \\
* & \\
* & \\
& a_{m m}
\end{array}\right]
$$

where $\operatorname{per}(A)=a_{11} \cdots a_{m m} \neq 0$. Since permutations of the rows and columns of $A$ induce in a natural way permutations of the rows and columns of $A \times B$, we may assume $A$ has the form (3.3). From this it follows equality occurs in (3.1).

Conversely, suppose both $A$ and $B$ have at least two nonzero permutations products. Since permutations of the rows and columns of $A$ and $B$ give rise to permutations of the rows and columns of $A \times B$, we may assume by Theorem 2.3 that 


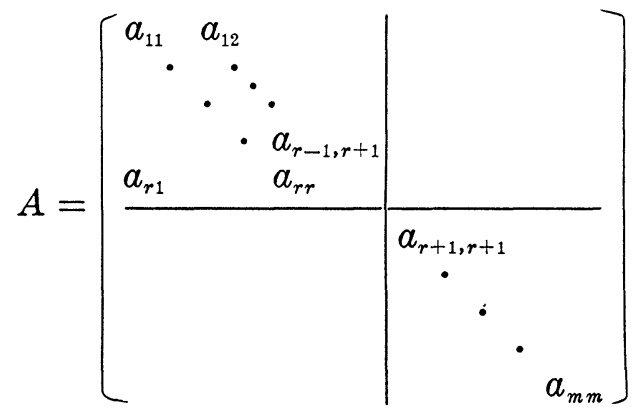

and

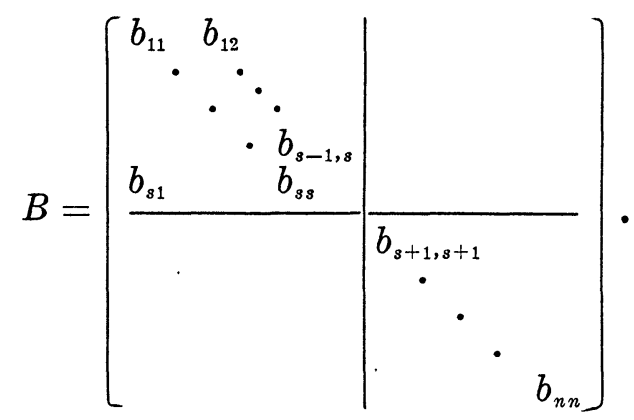

Here $2 \leqq r \leqq m$ and $2 \leqq s \leqq n$. Each $a_{i j}$ and $b_{k l}$ designated is not zero while all other entries not designated are arbitrary. Consider the matrices $A \times I_{n}$ and $I_{m} \times B$. Cross out column one of $A \times I_{n}$ to obtain the matrix $\left(A \times I_{n}\right)_{1}$ and cross out row one of $I_{m} \times B$ to obtain the matrix $\left(I_{m} \times B\right)_{1}$. The matrix $\left(A \times I_{n}\right)_{1}\left(I_{m} \times B\right)_{1}$ is of order $m n$ and we consider it to be partitioned into $m^{2}$ blocks (submatrices) of size $n$ by $n$ in the natural way. Just as in the direct product we shall speak of the $(i, j)$-block of $\left(A \times I_{n}\right)_{1}\left(I_{m} \times B\right)_{1}, 1 \leqq i, j \leqq m$. In each of the $(k, k)$-blocks, $k=1, \cdots, r-1$ select the last $n-1$ main diagonal elements; in the $(r, r)$-block select the elements in positions $(1,2), \cdots,(s-1, s),(s+1, s+1), \cdots,(n, n)$; in each of the $(j, j+1)-$ blocks, $j=1, \cdots, r-1$ select the first main diagonal element; in the $(r, 1)$-block select the element in position $(s, 1)$; and finally in each the $(i, i)$-blocks, $i=r+1, \cdots, n$ select all of the main diagonal elements. It can be verified that each of the elements selected is different from zero and that the collection form a permutation array of $\left(A \times I_{n}\right)_{1}\left(I_{m} \times B\right)_{1}$. Hence their product is a nonzero permutation product of $\left(A \times I_{n}\right)_{1}\left(I_{m} \times B\right)_{1}$ and

$$
\operatorname{per}\left(\left(A \times I_{n}\right)_{1}\left(I_{m} \times B\right)_{1}\right)>0 .
$$

By Theorem 2.4 strict inequality occurs in (3.2) and thus in (3.1). This concludes the proof of the theorem.

Corollary 3.2. Suppose both $A$ and $B$ have nonzero permanents. 
Then equality occurs in (3.1) if and only if by permuting rows and columns $A$ or $B$ can be brought to triangular form with nonzero elements on the main diagonal and zeros above the main diagonal.

Proof. This is a direct consequence of Theorems 2.1 and 3.1.

CoROLlaRY 3.3. If $A$ and $B$ are matrices of 0 's and 1's, then equality occurs in (3.1) if and only if per $(A)=0$ or 1 or $\operatorname{per}(B)=$ 0 or 1.

Theorem 3.1 may be generalized to include the direct product of any finite number matrices in the following way.

TheOREM 3.4. Let $A_{1}, A_{2}, \cdots, A_{n}$ be nonnegative matrices of orders $m_{1}, m_{2}, \cdots, m_{n}$ respectively, and let

$$
e_{i}=\prod_{\substack{j=1 \\ j \neq i}}^{n} m_{j} \quad(i=1,2, \cdots, n) .
$$

Then

(3.6) $\operatorname{per}\left(A_{1} \times A_{2} \times \cdots \times A_{n}\right) \geqq\left(\operatorname{per}\left(A_{1}\right)\right)^{e_{1}}\left(\operatorname{per}\left(A_{2}\right)\right)^{e_{2}} \cdots\left(\operatorname{per}\left(A_{n}\right)\right)^{e_{n}}$.

Equality occurs in (3.6) if and only if per $\left(A_{i}\right)=0$ for some $i=$ $1,2, \cdots, n$ or $(n-1)$ of the matrices $A_{1}, A_{2}, \cdots, A_{n}$ have exactly one nonzero permutation product.

Proof. Inequality (3.6) follows from Theorem 3.1 and an obvious induction on $n$. Suppose per $\left(A_{i}\right)=0$ for some $i=1,2, \cdots, n$. Using the fact that the direct product operation is associative and (1.4.), we may assume that per $\left(A_{n}\right)=0$. Then by Theorem 3.1 we obtain per $\left(A_{1} \times A_{2} \times \cdots A_{n}\right)=0$ and equality occurs in (3.6). Suppose $n-1$ of the matrices $A_{1}, A_{2}, \cdots, A_{n}$ have precisely one nonzero permutation product. By associativity and (1.4) we may assume $A_{1}, A_{2}, \cdots, A_{n-1}$ do. Then $A_{1} \times A_{2} \times \cdots \times A_{n-1}$ also has exactly one nonzero permutation product. Applying Theorem 3.1 we obtain equality in (3.6).

Conversely suppose $A_{1}, A_{2}, \cdots, A_{n}$ all have nonzero permanents and at least two have more than one nonzero permutation product. By associativity, (1.3), and (1.4) we may assume $A_{1}$ and $A_{2}$ do. Then by theorem 3.1 we have

$$
\begin{aligned}
\operatorname{per}\left(A_{1} \times A_{2}\right. & \left.\times \cdots \times A_{n}\right) \\
& \geqq\left(\operatorname{per}\left(A_{1} \times A_{2}\right)\right)^{m_{3} \cdots m_{n}}\left(\operatorname{per}\left(A_{3} \times \cdots \times A_{n}\right)\right)^{m_{1} m_{2}} \\
& >\left(\operatorname{per}\left(A_{1}\right)\right)^{e_{1}} \operatorname{per}\left(A_{2}\right)^{e_{2}}\left(\operatorname{per}\left(A_{3} \times \cdots \times A_{n}\right)\right)^{m_{1} m_{2}} \\
& \geqq\left(\operatorname{per}\left(A_{1}\right)\right)^{e_{1}}\left(\operatorname{per}\left(A_{2}\right)\right)^{e_{2}} \cdots\left(\operatorname{per}\left(A_{n}\right)\right)^{e_{n}}
\end{aligned}
$$


This establishes the theorem.

Inequality (3.1) and the more general (3.6) containing many inequalities obtained by specializing the matrices concerned. For instance if in (3.1) we let $A=J_{m}$, the matrix of 1's of order $m$, and $B=J_{n}$, the matrix of 1's of order $n$, we obtain

$$
(m n) ! \geqq(m !)^{n}(n !)^{m}
$$

Equality occurs in (3.7) if and only if $m=1$ or $n=1$.

The following theorem is basic.

THEOREM 3.5. Let $A$ and $B$ be matrices of orders $m$ and $n$ respectively with entries from a field $F$. Then every permutation product of $A \times B$ is expressible (in general, not uniquely) as the product of $n$ permutation products of $A$ and $m$ permutation products of $B$. Conversely, the product of $n$ permutation products of $A$ and $m$ permutation products of $B$ is a permutation product of $A \times B$.

Proof. Consider an arbitrary permutation product of $A \times B$ and a permutation array which gives rise to this product. Suppose this permutation array contains $c_{i j}$ entries from the $(i, j)$-block of $A \times B$, $1 \leqq i, j \leqq m$. Form the matrix

$$
C=\left[c_{i j}\right] \quad(i, j=1,2, \cdots, m) .
$$

$C$ is a matrix of order $m$ whose entries are nonnegative integers. Since $A$ and $B$ are square matrices, we have

$$
\sum_{j=1}^{m} c_{i j}=n \quad(i=1,2, \cdots, m),
$$

and

$$
\sum_{i=1}^{m} c_{i j}=n \quad(j=1,2, \cdots, m) .
$$

Then by $[3$, Th. 5.2, p. 56] we have

$$
C=c_{1} P_{1}+c_{2} P_{2}+\cdots+c_{t} P_{t}
$$

where $c_{1}, c_{2}, \cdots, c_{t}$ are positive integers with $c_{1}+c_{2}+\cdots c_{t}=n$ and where $P_{1}, P_{2}, \cdots, P_{t}$ are distinct permutation matrices of order $m$. Each permutation matrix $P_{k}$ corresponds in a natural way to a permutation array of $A$. Let this array be denoted by

$$
a_{1 \sigma_{k^{(1)}}}, a_{2 \sigma_{k}(2)}, \cdots, a_{m \sigma_{k}(m)}
$$

where $\sigma_{k}(1), \sigma_{k}(2), \cdots, \sigma_{k}(m)$ is a permutation of $1,2, \cdots, m$. Then 
the $m n a$ 's that appear in the given permutation product of $A \times B$ can be arranged as:

$$
\left(a_{1 \sigma_{1}(1)} a_{2 \sigma_{1}(2)} \cdots a_{m \sigma_{1}(m)}\right)^{c_{1}} \cdots\left(a_{1 \sigma_{t}^{(1)}} a_{2 \sigma_{t}(2)} \cdots a_{m \sigma_{t}(m)}\right)^{c_{t}} .
$$

Since there exists a permutation matrix $P$ such that $P^{T}(A \times B) P=$ $B \times A$, it follows that the specified permutation array of $A \times B$ is also a permutation array of $B \times A$. Therefore in a similar manner the $b$ 's in the given permutation product of $A \times B$ can be expressed as the product of $m$ permutation products of $B$.

Conversely, it is easy to verify that the product of $n$ permutation products of $A$ is a permutation product of $A \times I_{n}$ and the product of $m$ permutation products of $B$ is a permutation product of $I_{m} \times B$. The matrix product

$$
\left(A \times I_{n}\right)\left(I_{m} \times B\right)=A \times B
$$

yields the desired permutation product of $A \times B$.

COROLlaRY 3.6. There exists a minimal positive real number $K_{m, n}$, depending only on $m$ and $n$, such that

$$
\operatorname{per}(A \times B) \leqq K_{m, n}(\operatorname{per}(A))^{n}(\operatorname{per}(B))^{m}
$$

for any two nonnegative matrices $A$ and $B$ of orders $m$ and $n$ respectively.

Proof. By the theorem all of the distinct terms in per $(A \times B)$ appear at least once in the product $(\operatorname{per}(A))^{n}(\operatorname{per}(B))^{m}$. Since there are in total $(m n)$ ! terms in per $(A \times B)$, we have

$$
\operatorname{per}(A \times B) \leqq(m n) !(\operatorname{per}(A))^{n}(\operatorname{per}(B))^{m}
$$

for all nonnegative matrices $A$ and $B$ of orders $m$ and $n$ respectively. This shows the existence of the constant $K_{m, n}$.

The constant $K_{m, n}$ is given by the equation

$$
K_{m, n}=\text { l.u.b. } \frac{\operatorname{per}(A \times B)}{(\operatorname{per}(A))^{n}(\operatorname{per}(B))^{m}}
$$

where the least upper bound is taken over all nonnegative matrices $A$ and $B$ of orders $m$ and $n$ respectively with nonzero permanents. The ratio

$$
\frac{\operatorname{per}(A \times B)}{(\operatorname{per}(A))^{n}(\operatorname{per}(B))^{m}}
$$


is homogeneous in the sense that if a row or column of $A$ or $B$ is multiplied by a positive real number then the ratio is unchanged. This allows one to assume $A$ and $B$ are row stochastic (the sum of the elements of each row is one) in determining $K_{m, n}$. Also by continuity considerations only positive matrices $A$ and $B$ need be considered. Hence we may replace the l.u.b. in (3.8) by the l.u.b. over all positive row stochastic matrices $A$ and $B$ of orders $m$ and $n$ respectively. If in (3.8) we let $A=J_{m}$, the matrix of 1's of order $m$, and $B=J_{n}$, the matrix of 1's or order $n$, we obtain

$$
K_{m, n} \geqq \frac{(m n) !}{(m !)^{n}(n !)^{m}} .
$$

We conjecture here that (3.9) is actually an equality and therefore that

$$
\operatorname{per}(A \times B) \leqq \frac{(m n) !}{(m !)^{n}(n !)^{m}}(\operatorname{per}(A))^{n}(\operatorname{per}(B))^{m}
$$

for all nonnegative matrices $A$ and $B$ of orders $m$ and $n$ respectively. There is limited evidence to suggest that this is true. For instance it can be verified for $m=n=2$, i.e. $K_{2,2}=3 / 2$.

To conclude this section we give the following interpretation of Theorem 3.5. Let $S_{1}, S_{2}, \cdots, S_{m}$ be $m$ subsets of the elements $a_{1}, a_{2}, \cdots, a_{m}$ and $T_{1}, T_{2}, \cdots, T_{n}$ be $n$ subsets of the elements $b_{1}, b_{2}, \cdots, b_{n}$. Form the incidence matrices $A=\left[a_{i j}\right]$ and $B=\left[b_{k l}\right]$ of orders $m$ and $n$ respectively. Here $a_{i j}=1$ if $a_{j}$ is a member of $S_{i}$ and 0 otherwise. Similarly for $B$. For $i=1,2, \cdots, m$ and $j=1,2, \cdots, n$ define $S_{i} \times T_{j}$ to be a subset of all the ordered pairs

$$
\left(a_{r}, b_{s}\right) \quad(r=1,2, \cdots, m ; s=1,2, \cdots, n) .
$$

We have $\left(a_{r}, b_{s}\right)$ is a member of $S_{i} \times T_{j}$ if and only if $a_{r}$ is a member of $S_{i}$ and $b_{s}$ is a member of $T_{j}$. The incidence matrix of this collection of subsets is $A \times B$. Theorem 3.5 applied to this situation says that if we have a system of distinct representatives (SDR) of the collection of subsets

$$
S_{\imath} \times T_{j} \quad(i=1,2, \cdots, m ; j=1,2, \cdots n),
$$

then the first components of the members of this SDR can be arranged into $n$ SDR's of the collection $S_{1}, S_{2}, \cdots, S_{m}$ and the second components can be arranged into $m$ SDR's of the collection $T_{1}, T_{2}, \cdots, T_{n}$. Conversely, $n$ SDR's of $S_{1}, S_{2}, \cdots, S_{m}$ and $m$ SDR's of $T_{1}, T_{2}, \cdots, T_{n}$ can be paired up in at least one way to form an SDR of the collection of subsets in (3.10). 


\section{REFERENCES}

1. F.R. Gantmacher, The Theory of Matrices, vol. 2, New York, Chelsea, 1959.

2. C.C. MacDuffee, The Theory of Matrices, rev. ed., New York, Chelsea, 1946.

3. H.J. Ryser, Combinatorial Mathematics, Carus Math. Monograph, no. 14, New York, Wiley, 1963.

SYRACUSE UNIVERSITY 


\section{PACIFIC JOURNAL OF MATHEMATICS}

\section{EDITORS}

\author{
H. SAMmLSON \\ Stanford University \\ Stanford, California \\ R. M. BLUMENTHAL \\ University of Washington \\ Seattle, Washington 98105
}

\author{
*J. DUGUNDJI \\ University of Southern California \\ Los Angeles, California 90007
}

RICHARD ARENS

University of California

Los Angeles, California 90024

\section{E. F. BECKENBACH \\ B. H. NEUMANN \\ ASSOCIATE EDITORS}

\section{SUPPORTING INSTITUTIONS}

\author{
UNIVERSITY OF BRITISH COLUMBIA \\ CALIFORNIA INSTITUTE OF TECHNOLOGY \\ UNIVERSITY OF CALIFORNIA \\ MONTANA STATE UNIVERSITY \\ UNIVERSITY OF NEVADA \\ NEW MEXICO STATE UNIVERSITY \\ OREGON STATE UNIVERSITY \\ UNIVERSITY OF OREGON \\ OSAKA UNIVERSITY \\ UNIVERSITY OF SOUTHERN CALIFORNIA
}

\author{
STANFORD UNIVERSITY \\ UNIVERSITY OF TOKYO \\ UNIVERSITY OF UTAH \\ WASHINGTON STATE UNIVERSITY \\ UNIVERSITY OF WASHINGTON \\ * * * \\ AMERICAN MATHEMATICAL SOCIETY \\ CHEVRON RESEARCH CORPORATION \\ TRW SYSTEMS \\ NAVAL ORDNANCE TEST STATION
}

\footnotetext{
Mathematical papers intended for publication in the Pacific Journal of Mathematics should be typewritten (double spaced). The first paragraph or two must be capable of being used separately as a synopsis of the entire paper. It should not contain references to the bibliography. Manu. scripts may be sent to any one of the four editors. All other communications to the editors should be addressed to the managing editor, Richard Arens at the University of California, Los Angeles, California 90024.

50 reprints per author of each article are furnished free of charge; additional copies may be obtained at cost in multiples of 50 .
}

The Pacific Journal of Mathematics is published monthly. Effective with Volume 16 the price per volume (3 numbers) is $\$ 8.00$; single issues, $\$ 3.00$. Special price for current issues to individual faculty members of supporting institutions and to individual members of the American Mathematical Society: $\$ 4.00$ per volume; single issues $\$ 1.50$. Back numbers are available.

Subscriptions, orders for back numbers, and changes of address should be sent to Pacific Journal of Mathematics, 103 Highland Boulevard, Berkeley 8, California.

Printed at Kokusai Bunken Insatsusha (International Academic Printing Co., Ltd.), No. 6, 2-chome, Fujimi-cho, Chiyoda-ku, Tokyo, Japan.

PUBLISHED BY PACIFIC JOURNAL OF MATHEMATICS, A NON-PROFIT CORPORATION

The Supporting Institutions listed above contribute to the cost of publication of this Journal, but they are not owners or publishers and have no responsibility for its content or policies.

* Paul A. White, Acting Editor until J. Dugundji returns. 


\section{Pacific Journal of Mathematics}

\section{Vol. 16, No. $3 \quad$ BadMonth, 1966}

Gert Einar Torsten Almkvist, Stability of linear differential equations with

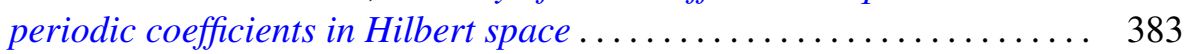

Richard Allen Askey and Stephen Wainger, A transplantation theorem for ultraspherical coefficients ................................ 393

Joseph Barback, Two notes on regressive isols .................. 407

Allen Richard Bernstein and Abraham Robinson, Solution of an invariant subspace problem of K. T. Smith and P. R. Halmos .............. 421

P. R. Halmos, Invariant subspaces of polynomially compact operators . . . . 433

Leon Bernstein, New infinite classes of periodic Jacobi-Perron algorithms.................................... 439

Richard Anthony Brualdi, Permanent of the direct product of matrices .... . 471

W. Wistar (William) Comfort and Kenneth Allen Ross, Pseudocompactness and uniform continuity in topological groups .................. 483

James Michael Gardner Fell, Algebras and fiber bundles . . . . . . . . . . . . 497

Alessandro Figà-Talamanca and Daniel Rider, A theorem of Littlewood and lacunary series for compact groups ..................... 505

David London, Two inequalities in nonnegative symmetric matrices...... 515

Norman Jay Pullman, Infinite products of substochastic matrices ........ 537

James McLean Sloss, Reflection and approximation by interpolation along

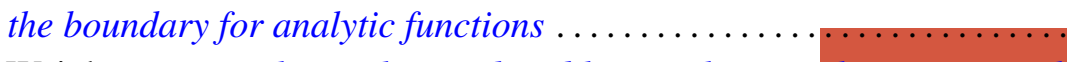

Carl Weinbaum, Visualizing the word problem, with an application to sixth groups................................... 